\title{
Pseudoconjugacy criteria for half-linear second order differential equations
}

\author{
Ondřej Došlý
}




\title{
PSEUDOCONJUGACY CRITERIA FOR HALF-LINEAR SECOND ORDER DIFFERENTIAL EQUATIONS
}

\author{
ONDŘEJ DOŠLÝ
}

\begin{abstract}
Aвstract. Using the variational principle, we present sufficient conditions for the existence of a pair of pseudoconjugate points in an interval $I \subseteq \mathbb{R}$ relative to the half-linear second order differential equation
\end{abstract}

$(*)$

$$
\left(r(t) \Phi\left(x^{\prime}\right)\right)^{\prime}+c(t) \Phi(x)=0, \quad \Phi(x)=|x|^{p-2} x, p>1 .
$$

The endpoints of the interval $I$ are allowed to be singular (in particular, the case $I=\mathbb{R}$ is possible) and an important role is played by the principal solution of $\left(^{*}\right)$ and of its reciprocal equation.

Mathematics Subject Classification: $34 \mathrm{C} 10$

Keywords: half-linear differential equation, pseudoconjugate points, pseudoconjugacy criterion, variational method, principal solution, reciprocal equation, coprincipal solution

\section{InTRODUCTION}

In this paper we deal with the half-linear second order differential equation

$$
\left(r(t) \Phi\left(x^{\prime}\right)\right)^{\prime}+c(t) \Phi(x)=0, \quad \Phi(x):=|x|^{p-2} x, p>1,
$$

where $r, c$ are continuous functions and $r(t)>0$ in an interval under consideration. Oscillation theory of (1) attracted considerable attention in recent years and many of the results of the classical linear oscillation theory (the linear Sturm-Liouville differential equation is a special case $p=2$ in (1)) have been extended to (1), see, e. g., [1, Chap. 3] and the references given therein.

Let $x$ be a nontrivial solution of (1) such that $x\left(t_{1}\right)=0$ for some $t_{1} \in \mathbb{R}$. A point $t_{2}>t_{1}$ is said to be the (right) conjugate point of $t_{1}$ (relative to (1)) if $x\left(t_{2}\right)=0$. A value $t_{2}>t_{1}$ is said to be the (right) pseudoconjugate point of $t_{1}$ (relative to (1)) if $x^{\prime}\left(t_{2}\right)=0$. Note that these definitions of (pseudo)conjugate points of $t_{1}$ are correct since by the homogeneity property of the solution space of (1), any nontrivial solution $z$ of (1) for which $z\left(t_{1}\right)=0$ is a constant multiple of $x$. Equation (1) is said

\footnotetext{
Research supported by grant A1163401/04 of the Grant Agency of the Czech Academy of Sciences and by the Research Project MSM0021622409 of Ministry of Education of the Czech Republic.
} 
to be (pseudo)conjugate on an interval $I$ if this interval contains at least one pair of (pseudo)conjugate points.

An integral comparison theorem for (pseudo)conjugate points relative to a pair of linear second order differential equations of the form

$$
\left(r(t) x^{\prime}\right)^{\prime}+c(t) x=0
$$

was established by Leighton in [8]. A part of this statement concerning conjugate points was extended to half-linear equation (1) by Jaroš and Kusano [7] and a "pseudoconjugate" part in the recent paper of Rostás [12]. These statements compare (1) with the equation of the same form

$$
\left(R(t) \Phi\left(y^{\prime}\right)\right)^{\prime}+C(t) \Phi(y)=0,
$$

and read as follows.

Proposition 1. Consider a pair of half-linear differential equations (1) and (2).

(i) Let $y$ be a nontrivial solution of (2) such that $y(a)=0=y(b)$, i. e., $b$ is $a$ right conjugate point of $t_{1}$. If

$$
\int_{a}^{b}\left\{[R(t)-r(t)]\left|y^{\prime}(t)\right|^{p}-[C(t)-c(t)]|y(t)|^{p}\right\} d t \leq 0,
$$

then any nontrivial solution of (1) with $x(a)=0$ has a zero point in $(a, b)$ or it is a constant multiple of $y$.

(ii) Let $y$ be a nontrivial solution of (2) such that $y(a)=0=y^{\prime}(b)$ and $y^{\prime}(t) \neq 0$ for $t \in[a, b), i . e ., b$ is the first right pseudoconjugate point of a relative to (2). If (3) holds, then any nontrivial solution $x$ of (1) with $x(a)=0$ has the property that $x^{\prime}(\xi)=0$ for some $\xi \in(a, b]$ with $\xi=b$ only if $x$ is a multiple of $y$.

Note that a closer examination of the proof of the previous statements reveals the fact that part (ii) can be reformulated as follows (part (i) can be reformulated in a similar way, but we will not need this modified statement in our paper).

Lemma 1. Suppose that there exists a piecewise differentiable function $z$ for which $z(a)=0=z^{\prime}(b)$ for $t \in[a, b)$ and

$$
\mathcal{F}_{r c}(z ; a, b):=\int_{a}^{b}\left[r(t)\left|z^{\prime}(t)\right|^{p}-c(t)|z(t)|^{p}\right] d t<0 .
$$

Then the nontrivial solution $x$ of (1) given by $x(a)=0$ satisfies $x^{\prime}(\xi)=0$ for some $\xi \in(a, b]$.

The aim of this paper is to investigate the situation when the endpoints $a, b$ are allowed to be singular points of the investigated equations. A typical example is $a=-\infty, b=\infty$ (but, of course, $a, b$ may also be finite singularities, i. e., points where the existence and uniqueness of solvability of the initial value problem for the investigated equations is violated). 
A singular version of Leighton's comparison theorem for conjugate points was established in several papers. It was discovered that the crucial role is played by the so-called principal solution of (half-)linear equations in these extensions.

As a classical paper concerning the linear Sturm-Liouville equation (1) is usually regarded the paper of Müller-Pfeiffer [10]. In that paper (and then in its improved version [11]) it is shown that under the assumptions

$$
\int^{\infty} \frac{d t}{r(t)}=\infty=\int_{-\infty} \frac{d t}{r(t)}
$$

equation (1) is conjugate on $\mathbb{R}$ provided

$$
\liminf _{t_{1} \downarrow-\infty, t_{2} \uparrow \infty} \int_{t_{1}}^{t_{2}} c(t) d t \geq 0 \quad \text { and } \quad c(t) \not \equiv 0 \text { on } \mathbb{R} .
$$

Observe that under (5) the function $\tilde{x}(t) \equiv 1$ is the principal solution of the one-term equation $\left(r(t) x^{\prime}\right)^{\prime}=0$ both at $-\infty$ and $\infty$ (for explanation see the next section) and that the integrand $c(t)$ in (6) can be written in the form $c(t) \tilde{x}^{2}(t)$ in this special case. This observation is also a link between (6) and the linear version of the part (i) of Proposition 1, since $y$ is the principal solution at (regular points) $a$ and $b$ of (2).

The half-linear extension of (6) is given in [4], where equation (1) is considered as a perturbation of the equation

$$
\left(r(t) \Phi\left(x^{\prime}\right)\right)^{\prime}+\tilde{c}(t) \Phi(x)=0 .
$$

It is supposed that (7) is disconjugate and its principal solutions at $a$ and $b$ coincide, i. e., $x_{a}=x_{b}=\tilde{x}$. Then $(1)$ is conjugate on $(a, b)$ provided

$$
\liminf _{t_{1} \downarrow a, t_{2} \uparrow b} \int_{t_{1}}^{t_{2}}[c(t)-\tilde{c}(t)] \tilde{x}^{p}(t) d t \geq 0, \quad c(t) \not \equiv \tilde{c}(t) \text { on }(a, b) .
$$

When $p=2,(a, b)=\mathbb{R}$ and $\tilde{c}(t) \equiv 0$, this conjugacy criterion reduces to (6). Concerning a singular version of part (ii) of Proposition 1, the main idea of our proof is based on the fact that the so-called coprincipal solution of (1) at $b$ plays the role of the solution satisfying $y^{\prime}(b)=0$ if $b$ is a singular point (and equals this solution if $b$ is a regular point).

The paper is organized as follows. In the next section we recall basic methods of the half-linear oscillation theory, including the concept of the principal solution of (1) which plays the fundamental role in our investigation. Section 3 contains the main results of the paper-two "singular" pseudoconjugacy criteria for (1), and the last section is devoted to examples which illustrate these criteria.

\section{Preliminaries}

In this section we recall some basic facts concerning equation (1). First of all let us mention that the linear oscillation theory extends verbatim to (1). In particular, the zero points of two linearly independent solutions of this equation separate each 
other and hence (1) can be classified as oscillatory or nonoscillatory at a singular point $b$ according to whether or not any solution has/does not have infinitely many zeros in any neighborhood of $b$. Similarly to the linear case, equation (1) cannot be oscillatory at a regular point since singular points are the only possible cluster points of a sequence of zero points of a solution of (1).

If $c(t) \neq 0$ in some interval $I$ and $x$ is a solution of (1), then $u=r \Phi\left(x^{\prime}\right)$ is a solution of the reciprocal equation

$$
\left(\frac{1}{\Phi^{-1}(c(t))} \Phi^{-1}\left(u^{\prime}\right)\right)^{\prime}+\frac{1}{r^{q-1}(t)} \Phi^{-1}(u)=0
$$

where $\Phi^{-1}(s)=|s|^{q-2} s$, is the inverse function of $\Phi, q$ being the conjugate number of $p$, i. e., $q=p /(p-1)$. The Rolle Mean Value theorem immediately implies that (1) is oscillatory if and only if the reciprocal equation (8) is oscillatory. If a solution $x$ satisfies $x(t) \neq 0$ in some interval $I$, then $w:=r \Phi\left(x^{\prime} / x\right)$ solves in $I$ the Riccati-type differential equation

$$
w^{\prime}+c(t)+(p-1) r^{1-q}(t)|w|^{q}=0 .
$$

Moreover, if $w(t) \neq 0$ for $t \in I$, then $v=-\frac{1}{\Phi^{-1}(w)}$ is a solution of the equation

$$
v^{\prime}+r^{1-q}(t)+(q-1) c(t)|v|^{p}=0
$$

which is the Riccati-type equation associated with reciprocal equation (8). We will also need the half-linear version of Picone's identity, which was established in [7]. We present here this identity in a modified form which is more suitable for our purpose.

Lemma 2. Let $w$ be a solution of Riccati-type equation (9), which exists for $t \in$ $[a, b]$. Then for any piecewise $C^{1}$ function $y$ we have

$$
\begin{aligned}
\int_{a}^{b}\left[r(t)\left|y^{\prime}\right|^{p}-c(t)|y|^{p}\right] d t=\left.w(t)|y|^{p}\right|_{a} ^{b} & \\
& +p \int_{a}^{b} r^{1-q}(t) P\left(r^{q-1}(t) y^{\prime}, w(t) \Phi(y)\right) d t
\end{aligned}
$$

where

$$
P(u, v):=\frac{|u|^{p}}{p}-u v+\frac{|v|^{q}}{q} \geq 0
$$

with equality only if $v=\Phi(u)$.

The crucial role in our investigation is played by the concept of the principal solution of nonoscillatory half-linear equation (1). This concept was introduced by Mirzov [9] as follows. We describe this construction near the singular point $b=\infty$ as it is done in Mirzov's original paper, but this construction extends also when $b$ is a finite singularity (or even a regular point). Suppose that (1) is nonoscillatory at $\infty$, i. e., there exists a solution $\hat{w}$ of associated Riccati-type equation (9) which is defined 
on an interval $[T, \infty)$. Let $d>T$ and $x_{d}$ be the solution of (1) given by the initial condition $x_{d}(d)=0, x_{d}^{\prime}(d)=-1$ and let $w_{d}=r \Phi\left(x_{d}^{\prime} / x_{d}\right)$. Then for $T<d_{1}<d_{2}$ we have $\hat{w}(t)>w_{d_{1}}(t)>w_{d_{2}}(t)$ for $t \in\left[T, d_{1}\right)$. This means that on every compact subinterval of $[T, \infty)$ there exists the uniform limit

$$
\tilde{w}(t):=\lim _{d \rightarrow \infty} w_{d}(t)
$$

and hence this function is a solution of (9). This solution is called the eventually minimal solution of (9). The corresponding solution $\tilde{x}$ of (1) given by the formula

$$
\tilde{x}(t)=K \exp \left\{\int_{T}^{t} \Phi(\tilde{w}(s) / r(s))\right\} d s,
$$

where $K$ is a nonzero real constant, is called the principal solution (at $\infty$ ) of (1). Note that the largest zero point $\tilde{t}$ of the principal solution $\tilde{x}$ (if exists) plays essentially the role of the first left conjugate point of $t=\infty$ since any solution $x$ of (1) linearly independent of $\tilde{x}$ has exactly one zero in $(\tilde{t}, \infty)$, see [7]. Note also that if $t=b$ is a regular point of (1), then the principal solution at $b$ is simply defined as a nontrivial solution satisfying $x(b)=0$. Finally, a solution $\tilde{x}$ of nonoscillatory equation (1) is said to be coprincipal at $b$, if its quasiderivative $\tilde{u}=r \Phi\left(\tilde{x}^{\prime}\right)$ is the principal solution at $b$ of the reciprocal equation (8). In the last two statements of this section we recall some properties of the principal and coprincipal solutions of (1). The first one is formulated in [5] in case $b=\infty$, but a closer examination of its proof reveals that the statement remains to hold also when $b$ is a finite singularity.

Lemma 3. Suppose that $\int^{b} r^{1-q}(t) d t=\infty, c(t)>0$ near $b$ and (1) is nonoscillatory at $b$.

(i) If $\tilde{x}$ is the principal solution of equation (1) at b, then it is also a coprincipal solution at $b$.

(ii) A solution $\tilde{x}$ of (1) is principal at $b$ if and only if

$$
\int^{b} \frac{d t}{r(t) \tilde{x}^{2}(t)\left|\tilde{x}^{\prime}(t)\right|^{p-2}}=\infty
$$

The following alternative integral characterization of the principal solution of (1) is proved in the recent paper [3]. Note that this paper actually formulates the statement with the power $x^{2}$ instead of $x^{p}$ in the formula (14) given below. However, a closer examination of the proof given in [3] reveals that the statement remains to hold in this modified setting which better fits our treatment.

Lemma 4. Suppose that $c(t)>0$ for large $t$, equation (1) is nonoscillatory and both integrals $\int^{\infty} r^{1-q}(t) d t$ and $\int^{\infty} c(t) d t$ are convergent. Then $x$ is the principal solution of (1) if and only if

$$
\int^{\infty} \frac{d t}{r^{q-1}(t) x^{p}(t)}=\infty
$$




\section{EXISTENCE OF PSEUdOCONJUGATE POINTS}

Our main result reads as follows.

Theorem 1. Suppose that (2) is disconjugate on the interval $I=(a, b),-\infty \leq a<$ $b \leq \infty, C(t) \geq c(t)>0$ and $0<R(t) \leq r(t)$ near $a$ and $b$. Further, let $y$ be the positive principal solution of (2) at a and suppose that this solution is coprincipal at $b$ and $y^{\prime}(t)>0$ for $t \in(a, b)$. If

$$
\limsup _{t_{1} \downarrow a, t_{2} \uparrow b} \mathcal{J}\left(y ; t_{1}, t_{2}\right)<0,
$$

where

$$
\mathcal{J}\left(y ; t_{1}, t_{2}\right):=\int_{t_{1}}^{t_{2}}\left[(R(t)-r(t)) y^{\prime p}(t)-(C(t)-c(t))\right] y^{p}(t) d t
$$

then (1) is pseudoconjugate on I, i. e., there exists $a<d_{1}<d_{2}<b$ and a nontrivial solution $x$ of (1) such that $x\left(d_{1}\right)=0=x^{\prime}\left(d_{2}\right)$.

Proof. Let $a<t_{0}<t_{1}<t_{2}<t_{3}<b$ (these values will be specified later), and define a piecewise differentiable function $z$ for which $z\left(t_{0}\right)=0=z^{\prime}\left(t_{3}\right)$ as follows:

$$
z(t)= \begin{cases}0 & \text { for } t \in\left(a, t_{0}\right], \\ f(t) & \text { for } t \in\left[t_{0}, t_{1}\right], \\ y(t) & \text { for } t \in\left[t_{1}, t_{2}\right], \\ g(t) & \text { for } t \in\left[t_{2}, t_{3}\right], \\ g\left(t_{3}\right) & \text { for } t \in\left[t_{3}, b\right) .\end{cases}
$$

Here, $f$ is a solution of (2) satisfying the boundary conditions

$$
f\left(t_{0}\right)=0, \quad f\left(t_{1}\right)=y\left(t_{1}\right),
$$

this solution exists in view of disconjugacy of (1). The function $g$ is also the solution of (2), given by the boundary condition

$$
g\left(t_{2}\right)=y\left(t_{2}\right), \quad g^{\prime}\left(t_{3}\right)=0,
$$

i. e., the quasiderivative $u_{g}=R \Phi\left(g^{\prime}\right)$ is a solution of the reciprocal equation to (2)

$$
\left(C^{1-q}(t) \Phi^{-1}\left(u^{\prime}\right)\right)^{\prime}+R^{1-q}(t) \Phi^{-1}(u)=0
$$

satisfying the boundary conditions

$$
u_{g}^{\prime}\left(t_{2}\right)=-C\left(t_{2}\right) \Phi\left(y\left(t_{2}\right)\right), \quad u_{g}\left(t_{3}\right)=0 .
$$

The existence of this solution we prove as follows. The solution $w=R \Phi\left(y^{\prime} / y\right)$ of the Riccati-type differential equation associated with (2) is positive on $(a, b)$ (since $y^{\prime}(t)>0$ on $\left.(a, b)\right)$ and hence the solution $v=-\left(1 / \Phi^{-1}(w)\right)$ of the Riccati-type equation corresponding to the reciprocal equation (18) is negative on $(a, b)$. Now, 
let $u$ be a solution of reciprocal equation (18) for which $u\left(t_{3}\right)=0$ and suppose that $u^{\prime}(s)=0$ for some $a<s<t_{3}<b$. Then integration by parts yields

$$
\begin{aligned}
\int_{s}^{t_{3}}\left[C^{1-q}(t)\left|u^{\prime}\right|^{p}-R^{1-q}(t)|u|^{p}\right] d t & =\left.C^{1-q} u \Phi^{-1}\left(u^{\prime}\right)\right|_{s} ^{t_{3}} \\
& -\int_{s}^{t_{3}} u\left[\left(C^{1-q}(t) \Phi^{-1}\left(u^{\prime}\right)\right)^{\prime}+R^{1-q}(t) \Phi^{-1}(u)\right] d t=0
\end{aligned}
$$

and, at the same time, applying the Picone identity to the above integral we get

$$
\begin{aligned}
\int_{s}^{t_{3}}\left[C^{1-q}(t)\left|u^{\prime}\right|^{p}-R^{1-q}(t)|u|^{p}\right] d t & =-v(s)|u(s)|^{p} \\
& +q \int_{s}^{t_{3}} C(t) P\left(v \Phi^{-1}(u), C(t) u^{\prime}\right) d t>0,
\end{aligned}
$$

a contradiction. Hence the solution $u$ satisfies $u^{\prime}(t) \neq 0$ for $t<t_{3}$. Consequently, multiplying this solution by a suitable constant, we get the solution satisfying (19).

In the remaining part of the proof we will show that $\mathcal{F}\left(z ; t_{0}, t_{3}\right)<0$ if $t_{0}, t_{3}$ are sufficiently close to $a, b$, respectively, which then means that (1) is pseudoconjugate on $\left[t_{0}, t_{3}\right] \subset(a, b)$ by Lemma 1 . Using integration by parts, we get

$$
\begin{aligned}
\mathcal{F}_{R C}\left(f ; t_{0}, t_{1}\right) & :=\int_{t_{0}}^{t_{1}}\left[R(t)\left|f^{\prime}(t)\right|^{p}-C(t)|f(t)|^{p}\right] d t \\
& =\left.R(t) f(t) \Phi\left(f^{\prime}(t)\right)\right|_{t_{0}} ^{t_{1}}-\int_{t_{0}}^{t_{1}} f(t)\left[\left(R(t) \Phi\left(f^{\prime}(t)\right)^{\prime}+C(t) \Phi(f(t))\right] d t\right. \\
& =f^{p}\left(t_{1}\right) w_{f}\left(t_{1}\right),
\end{aligned}
$$

where $w_{f}=R \Phi\left(f^{\prime} / f\right)$. Similarly,

$$
\begin{aligned}
& \mathcal{F}_{R C}\left(y, t_{1}, t_{2}\right)=y^{p}\left(t_{2}\right) w_{y}\left(t_{2}\right)-y^{p}\left(t_{1}\right) w_{y}\left(t_{1}\right), \quad w_{y}=\frac{R \Phi\left(y^{\prime}\right)}{y}, \\
& \mathcal{F}_{R C}\left(g ; t_{2}, t_{3}\right)=-g^{p}\left(t_{2}\right) w_{g}\left(t_{2}\right), \quad w_{g}=\frac{R \Phi\left(g^{\prime}\right)}{\Phi(g)} .
\end{aligned}
$$

Summarizing these computations and taking into account (16), (17), we obtain

$$
\mathcal{F}_{R C}(z ; a, b)=\left(w_{f}-w_{y}\right)\left(t_{1}\right) y^{p}\left(t_{1}\right)+\left(w_{y}-w_{g}\right)\left(t_{2}\right) y^{p}\left(t_{2}\right) .
$$

From the definition of the principal solution at $a$, we have

$$
w_{f}\left(t_{1}\right) \rightarrow w_{y}\left(t_{1}\right) \quad \text { as } \quad t_{0} \rightarrow a+.
$$

Note that $w_{f}$ depends on $t_{0}$. Concerning the difference $w_{y}\left(t_{2}\right)-w_{g}\left(t_{2}\right)$, the functions $v_{y}=-\left(1 / \Phi^{-1}\left(w_{y}\right)\right)$ and $v_{g}=-\left(1 / \Phi^{-1}\left(w_{g}\right)\right)$ are solutions of the Riccati equation (10) associated with reciprocal equation (8), and again $v_{g}\left(t_{2}\right) \rightarrow v_{y}\left(t_{2}\right) \neq 0$ as $t_{3} \rightarrow b-$, 
since we suppose that $y$ is the coprincipal solution at $b$, i. e., $v_{y}$ is the eventually minimal solution of (10) at $b$. Hence also

$$
w_{g}\left(t_{2}\right) \rightarrow w_{y}\left(t_{2}\right) \quad \text { as } \quad t_{2} \rightarrow b-
$$

Summarizing the previous computations and using inequalities between $R, r$ and $C, c$ near $a$ and $b$, we have

$$
\begin{aligned}
\mathcal{F}_{r c}\left(z ; t_{0}, t_{3}\right) & \leq \mathcal{F}_{R C}\left(z ; t_{0}, t_{3}\right)+\mathcal{J}\left(y ; t_{1}, t_{2}\right) \\
& =\left(w_{f}-w_{y}\right)\left(t_{1}\right) y^{p}\left(t_{1}\right)+\left(w_{y}-w_{g}\right)\left(t_{2}\right) y^{p}\left(t_{2}\right)+\mathcal{J}\left(y ; t_{1}, t_{2}\right) .
\end{aligned}
$$

Now, let $\varepsilon>0$ be such that limsup in (15) is less than $-4 \varepsilon$, i. e., $\mathcal{J}\left(y ; t_{1}, t_{3}\right)<-3 \varepsilon$ if $t_{1}, t_{2}$ are sufficiently close to $a$ and $b$, respectively. According to (20) and (21), if $t_{0}<t_{1}, t_{3}>t_{2}$ are sufficiently close to $a$ and $b$, respectively, we have

$$
\left|\left(w_{f}-w_{y}\right)\left(t_{1}\right) y^{p}\left(t_{1}\right)\right|<\varepsilon, \quad\left|\left(w_{y}-w_{g}\right)\left(t_{2}\right) y^{p}\left(t_{2}\right)\right|<\varepsilon .
$$

Consequently, for $t_{0}<t_{1}<t_{2}<t_{3}$ chosen in the above specified way, we obtain

$$
\mathcal{F}_{r c}(z) \leq-3 \varepsilon+\varepsilon+\varepsilon<0 .
$$

Now the statement of the theorem follows from Lemma 1.

In the next statement we show that if the inequality $R(t) \leq r(t)$ holds on whole interval $(a, b)$ (and not only near $a$ and $b$ as supposed in Theorem 1 ), the statement of this theorem can be modified as follows.

Theorem 2. Suppose that the assumptions of Theorem 1 are satisfied, except for the assumption $C(t) \geq c(t)>0$ near $a$, and let $R(t) \leq r(t)$ for $t \in(a, b)$. If

$$
\liminf _{s_{1} \downarrow a, s_{2} \uparrow b} \int_{s_{1}}^{s_{2}}(c(t)-C(t)) y^{p}(t) d t \geq 0 \quad \text { and } \quad C(t) \not \equiv c(t) \text { for } t \in(a, b),
$$

then (1) is pseudoconjugate on I, i. e., there exist $a<d_{1}<d_{2}<b$ and a nontrivial solution $x$ of (1) such that $x\left(d_{1}\right)=0=x^{\prime}\left(d_{2}\right)$.

Proof. We use essentially the same function $z$ as defined in the proof of Theorem 1 , with the following modification. Continuity of the functions $C, c$ and (22) imply the existence of $\bar{t} \in\left(t_{1}, t_{2}\right)$ and $d, \varrho>0$ such that $(c(t)-C(t)) y^{p}(t)>d$ for $(\bar{t}-\varrho, \bar{t}+\varrho) \subset$ $\left(t_{1}, t_{2}\right)$. Let $\alpha$ be any positive differentiable function with the compact support in $(\bar{t}-\varrho, \bar{t}+\varrho)$. Now, we modify the function $z$ for $t \in\left[t_{1}, t_{2}\right]$ as follows:

$$
z(t)= \begin{cases}y(t) & \text { for } t \in\left[t_{1}, t_{2}\right] \backslash[\bar{t}-\varrho, \bar{t}+\varrho] \\ y(t)(1+\delta \alpha(t)) & \text { for } t \in[\bar{t}-\varrho, \bar{t}+\varrho]\end{cases}
$$


where $\delta>0$ will be specified later. With this modification, using Picone's identity from Lemma 2,

$$
\begin{gathered}
\mathcal{F}_{R C}\left(z ; t_{1}, t_{2}\right)=\int_{t_{1}}^{t_{2}}\left[R(t)\left|z^{\prime}\right|^{p}-C(t) z^{p}\right] d t=\left.w_{y} z^{p}\right|_{t_{1}} ^{t_{2}} \\
+\int_{t_{1}}^{t_{2}}\left[R(t)\left|z^{\prime}\right|^{p}-p w_{y}(t) z^{\prime} \Phi(z)+(p-1) R^{1-q}(t)\left|w_{y}(t)\right|^{q} z^{p}\right] d t \\
=\left.w_{y} y^{p}\right|_{t_{1}} ^{t_{2}}+\int_{\bar{t}-\varrho}^{\bar{t} \varrho \varrho}\left\{R(t)\left|y^{\prime}+\delta(\alpha y)^{\prime}\right|^{p}-p R(t) \frac{\Phi\left(y^{\prime}\right)}{\Phi(y)} z^{\prime} y^{p-1}(1+\delta \alpha)^{p-1}\right. \\
\left.+(p-1) R^{1-q}(t)\left|\frac{R(t) \Phi\left(y^{\prime}\right)}{\Phi(y)}\right|^{q} y^{p}(1+\delta \alpha)^{p}\right\} d t \\
=\left.w_{y} y^{p}\right|_{t_{1}} ^{t_{2}}+\int_{\bar{t}-\varrho}^{\bar{t}+\varrho} R(t)\left\{\left|y^{\prime}\right|^{p}+p \delta(\alpha y)^{\prime} \Phi\left(y^{\prime}\right)+o(\delta)\right. \\
\left.-p\left(y^{\prime}+\delta(\alpha y)^{\prime}\right) \Phi\left(y^{\prime}\right)(1+(p-1) \delta \alpha+o(\delta))+(p-1)\left|y^{\prime}\right|^{p}(1+p \delta \alpha+o(\delta))\right\} d t \\
=\left.w_{y} y^{p}\right|_{t_{1}} ^{t_{2}}+\int_{\bar{t}-\varrho}^{\bar{t}+\varrho} R(t)\left\{\left|y^{\prime}\right|^{p}+p \delta(\alpha y)^{\prime} \Phi\left(y^{\prime}\right)+p\left|y^{\prime}\right|^{p}-p \delta \Phi\left(y^{\prime}\right)(\alpha y)^{\prime}\right. \\
\left.-p(p-1) \delta \alpha\left|y^{\prime}\right|^{p}+(p-1)\left|y^{\prime}\right|^{p}+(p-1) p \delta \alpha\left|y^{\prime}\right|^{p}+o(\delta)\right\} d t \\
\quad-\left.(p) w_{y} y^{p}\right|_{t_{1}} ^{t_{2}}+o(\delta) .
\end{gathered}
$$

Consequently, using the rechniques from the proof of Theorem 1, we get

$$
\begin{aligned}
\mathcal{F}_{R C}\left(z ; t_{0}, t_{3}\right)= & \int_{t_{0}}^{t_{3}}\left[R(t)\left|z^{\prime}\right|^{p}-C(t)|z|^{p}\right] d t \\
= & \left.w_{f}|f|^{p}\right|_{t_{0}} ^{t_{1}}+\left.w_{y} y^{p}\right|_{t_{1}} ^{t_{2}}+\left.w_{g}|g|^{p}\right|_{t_{2}} ^{t_{3}}+o(\delta) \\
= & y^{p}\left(t_{1}\right)\left(w_{f}\left(t_{1}\right)-w_{y}\left(t_{1}\right)\right) \\
& \quad+y^{p}\left(t_{2}\right)\left(w_{y}\left(t_{2}\right)-w_{g}\left(t_{2}\right)\right)+o(\delta)
\end{aligned}
$$

as $\delta \rightarrow 0+$. Concerning the interval $\left(t_{1}, t_{2}\right)$, we have

$$
\begin{gathered}
\int_{t_{1}}^{t_{2}}(c(t)-C(t)) z^{p}(t) d t=\int_{t_{1}}^{\bar{t}-\varrho}(c(t)-C(t)) y^{p}(t) \\
\quad+\int_{\bar{t}-\varrho}^{\bar{t}+\varrho}(c(t)-C(t)) y^{p}(t)(1+\delta \alpha(t))^{p} d t+\int_{\bar{t}+\varrho}^{t_{2}}(c(t)-C(t)) y^{p}(t) d t \\
=\int_{t_{1}}^{t_{2}}(c(t)-C(t)) y^{p}(t) d t+p \delta \int_{\bar{t}-\varrho}^{\bar{t}+\varrho}(c(t)-C(t)) y^{p}(t) \alpha(t) d t+o(\delta) \\
\geq \int_{t_{1}}^{t_{2}}(c(t)-C(t)) y^{p}(t) d t+\delta K+o(\delta),
\end{gathered}
$$


where $K=p d \int_{\bar{t}-\varrho}^{\bar{t} \varrho \varrho} \alpha(t) d t>0$. Therefore

$$
\int_{t_{1}}^{t_{2}}(c(t)-C(t)) z^{p}(t) t \geq \int_{t_{1}}^{t_{2}}(c(t)-C(t)) y^{p}(t) d t+K \delta+o(\delta) .
$$

Next we prove that the function $f / y$ is increasing on $\left(t_{0}, t_{1}\right)$. We have $(f / y)\left(t_{0}\right)=0$, $(f / y)\left(t_{1}\right)=1$ and $(f / y)^{\prime}(t) \neq 0$ for $t \in\left(t_{0}, t_{1}\right)$. Indeed, if $(f / y)^{\prime}(\tau)=0$ for some $\tau \in\left(t_{0}, t_{1}\right), w_{f}(\tau)=w_{h}(\tau)$ and this contradicts the unique solvability of the Riccati equation (9). Due to the monotonicity of $f / y$, the Second Mean Value Theorem of integral calculus implies that there exists $\xi \in\left(t_{0}, t_{1}\right)$ for which

$$
\begin{aligned}
\int_{t_{0}}^{t_{1}}[C(t)-c(t)] f^{p}(t) d t & =\int_{t_{0}}^{t_{1}}[C(t)-c(t)](f / y)^{p}(t) y^{p}(t) d t \\
& =\int_{\xi}^{t_{1}}[C(t)-c(t)] y^{p}(t) d t .
\end{aligned}
$$

Using the above computations and, again, the inequalities between the functions $r, R$ and $c, C$, we get

$$
\begin{aligned}
\mathcal{F}_{r c}\left(z ; t_{0}, t_{3}\right) \leq & \mathcal{F}_{R C}\left(z ; t_{0} ; t_{3}\right)-\int_{t_{0}}^{t_{2}}(c(t)-C(t)) z^{p}(t) d t \\
\leq & \left|w_{f}\left(t_{1}\right)-w_{y}\left(t_{1}\right)\right| y^{p}\left(t_{1}\right)+\left|w_{y}\left(t_{2}\right)-w_{g}\left(t_{2}\right)\right| y^{p}\left(t_{2}\right) \\
& \quad-\int_{\xi}^{t_{2}}(c(t)-C(t)) y^{p}(t) d t-K \delta+o(\delta) .
\end{aligned}
$$

Now, let $\delta>0$, a sufficiently small number, be such that $K \delta+o(\delta)=: \varepsilon>0$. According to (22) the points $t_{1}, t_{2}$ can be chosen in such a way that

$$
\int_{\xi}^{t_{2}}(c(t)-C(t)) y^{p}(t) d t>-\frac{\varepsilon}{4}
$$

whenever $\xi \in\left(a, t_{1}\right)$. Further, similarly as in the proof of Theorem 1

$$
y^{p}\left(t_{1}\right)\left|w_{f}\left(t_{1}\right)-w_{y}\left(t_{1}\right)\right|<\frac{\varepsilon}{4}, \quad y^{p}\left(t_{2}\right)\left|w_{y}\left(t_{2}\right)-w_{g}\left(t_{2}\right)\right|<\frac{\varepsilon}{4}
$$

if $t_{0}<t_{1}, t_{2}<t_{3}$ are sufficiently close to $a$ and $b$, respectively. Consequently, for the above specified choice of $t_{0}<t_{1}<t_{2}<t_{3}$ we have the inequality $\mathcal{F}_{r c}\left(z ; t_{0}, t_{3}\right)<$ $-(\varepsilon / 4)<0$ and Lemma 1 implies that $(1)$ is pseudoconjugate on $\left[t_{0}, t_{1}\right] \subset(a, b)$.

\section{EXAMPLeS}

We finish the paper with two examples illustrating the results of the previous section.

Example 1. Consider the half-linear Euler equation with the so-called critical coefficient

$$
\left(\Phi\left(x^{\prime}\right)\right)^{\prime}+\frac{\gamma_{p}}{t^{p}} \Phi(x)=0
$$


where $\gamma_{p}=(p-1)^{p} p^{-p}$. One solution of this equation can be computed explicitly if we look for this solution in the form $x(t)=t^{\lambda}$. Then substituting into (23) we find that this function is a solution of (23) if $\lambda$ is a root of the equation

$$
G(\lambda):=(p-1)\left(|\lambda|^{p}-\Phi(\lambda)\right)+\gamma_{p}=0,
$$

and by direct computation we have that $\lambda=(p-1) / p$ is a double root of $G$, hence $x(t)=t^{(p-1) / p}$ is a solution of (23). Using Lemma 3, it is not difficult to verify that $x$ is the principal solution at $t=0$, and principal as well as coprincipal solution at $\infty$. Consequently, equation (23) may play the role of equation (2) in Theorems 1, 2, and hence the equation

$$
\left(\Phi\left(x^{\prime}\right)\right)^{\prime}+\left(\frac{\gamma_{p}}{t^{p}}+c(t)\right) \Phi(x)=0
$$

with $c(t) \geq 0$ for $t \in(0, \delta), \delta>0$, and for large $t$, is pseudoconjugate on $(0, \infty)$ provided

$$
\liminf _{t_{1} \downarrow 0, t_{2} \uparrow \infty} \int_{t_{1}}^{t_{2}} c(t) t^{p-1} d t \geq 0 \quad \text { and } \quad c(t) \not \equiv 0, t \in(0, \infty) .
$$

Example 2. In this example we consider the half-linear differential equation

$$
\left(\left(1+t^{p}\right)^{p-1} \Phi\left(x^{\prime}\right)\right)^{\prime}+\frac{p-1}{1+t^{p}} \Phi(x)=0, \quad t \in[0, \infty) .
$$

By direct computation one can verify that

$$
x(t)=\frac{t}{\sqrt[p]{1+t^{p}}}, \quad u(t)=\left(1+t^{p}\right)^{p-1} \Phi\left(x^{\prime}(t)\right)=\frac{1}{\sqrt[q]{1+t^{p}}}
$$

are solutions of (24) and of its reciprocal equation

$$
\left(\left(1+t^{p}\right)^{q-1} \Phi^{-1}\left(u^{\prime}\right)\right)^{\prime}+\frac{(p-1)^{q-1}}{1+t^{p}} \Phi^{-1}(u)=0,
$$

respectively. Moreover, using Lemma 3 and Lemma 4 (applied to (26), i. e., with $p$ replaced by $q$ and vice versa) one can verify by a direct computation that the solution $x$ is principal at $t=0$ and coprincipal at $\infty$.

Since the point $t=0$ is the regular point of (24), the equation

$$
\left(\left(1+t^{p}\right)^{p-1} \Phi\left(x^{\prime}\right)\right)^{\prime}+\left(\frac{p-1}{1+t^{p}}+c(t)\right) \Phi(x)=0
$$

with $c(t) \geq 0$ for large $t$ is pseudoconjugate on $[0, \infty)$ provided

$$
\liminf _{t \rightarrow \infty} \int_{0}^{t} c(s) \frac{s^{p}}{1+s^{p}} d s \geq 0 \quad \text { and } \quad c(t) \not \equiv 0, t \in(0, \infty) .
$$




\section{REFERENCES}

[1] Agarwal, R. P., Grace, S. R., and O'Regan, D.: Oscillation Theory of Second Order Linear, Half-Linear, Superlinear and Sublinear Dynamic Equations, Kluwer AP, Dordrecht, etc., 2002.

[2] Ahlbrandt, C. D., Principal and antiprincipal solutions of self-adjoint differential systems and their reciprocals, Rocky Mountain J. Math., 2 (1972), 169-189.

[3] Cecchi, M., DošLá, Z., AND MARINI, I.: Half-linear differential equations and characteristic properties of principal solutions, J. Differential Equations, 208 (2005), 494-507.

[4] DošLÝ, O.: A remark on conjugacy of half-linear second order differential equations, Math. Slovaca, 50 (2000), 67-79.

[5] DošLÝ, O. AND Elbert, Á.: Integral characterization of principal solution of half-linear differential equations, Studia Sci. Math. Hungar., 36 (2000), 455-469.

[6] Elbert, Á. And Kusano, T: Principal solutions of nonoscillatory half-linear differential equations, Adv. Math. Sci. Appl., 18 (1998), 745-759.

[7] Jaroš, J. and Kusano, T., A Picone type identity for half-linear differential equations, Acta Math. Univ. Comenianea, 68 (1999), 137-151.

[8] Leighton, W:, Some elementary Sturm Theory, J. Differential Equations, 4 (1968), 187-193.

[9] Mirzov, J. D.: Principal and nonprincipal solutions of a nonoscillatory system, Tbiliss. Gos. Univ. Inst. Prikl. Mat. Trudy, 31 (1988), 100-117.

[10] Müller-PFeIfFer, E.: Existence of conjugate points for second and fourth order differential equations, Proc. Roy. Soc. Edinburgh, 89A (1981), 281-291.

[11] Müller-PfeifFer, E.: Nodal domains of one- or two-dimensional elliptic differential equations, Z. Anal. Anwendungen, 7 (1988), 135-139.

[12] Rostás, K.: Comparison theorems for pseudoconjugate points of half-linear ordinary differential equations of the second order, Acta Math. Univ. Comenianea, 72 (2003), 223-228.

\section{Author's address}

\section{Ondřej Došlý:}

Department of Mathematics, Masaryk University, Janáč́ovo nám. 2A, 66295 Brno, Czech RepubLIC.

E-mail address: dosly@math.muni.cz 